109 OVEREXPRESSION OF ENDOTHELIAL INSULIN-LIKE
GROWTH FACTOR-1 RECEPTORS (IGF-1R): A NOVEL ROLE
FOR IGF-1R IN ENDOTHELIAL FUNCTION AND REPAIR

doi:10.1136/heartjnl-2012-301877b.109

H Imrie, * H Viswambharan, P Sukumar, A Abbas, N Yuldasheva, M Gage, S Galloway, S B Wheatcroft, M T Kearney. University of Leeds, Leeds, UK

Introduction Endothelium-derived nitric oxide (NO) is a critical regulator of vascular homeostasis, repair and regeneration. We recently demonstrated that reducing insulin-like growth factor-1 receptor (IGF-1R) numbers in the endothelium, thereby decreasing the proportion of insulin resistant hybrid receptors, enhances NO bioavailability and increases endothelial cell (EC) insulin sensitivity (Abbas*, Imrie*, Viswambharan* et al. Diabetes 2011;60:2169-78). Methods To examine the effect of increasing IGF-1R in EC we generated transgenic mice overexpressing the human IGF-1R in EC (human IGF-1R endothelium overexpressing mice (hIGFREO)). Glucose and insulin sensitivity were measured by tolerance testing and plasma insulin and IGF-1 levels were analysed by ELISA. The response of aortic rings to increasing doses of phenylepherine (PE), with and without L-NMMA (a NO inhibitor), were measured ex vivo in an organ bath. NO release, eNOS activity and phosphorylation of eNOS (all in response to insulin) were measured by DAF fluorescence, the conversion of L-arginine to L-citrulline, and western blotting respectively. Endothelial regeneration was investigated by guide-wire injury of the femoral artery with quantification of Evans-blue dyed denuded area and migration assays were performed in response to VEGF by H\&E staining.

Results Increased endothelial IGF-1R numbers had no effect on glucose tolerance or insulin sensitivity in hIGFREO mice compared to wild-type (wt) littermates and fasting plasma glucose, insulin and IGF-1 levels were similar. Aortae from hIGFREO mice were hypercontractile to phenylephrine (PE) (Emax wt $=0.62 \pm 0.045$ vs hIGFREO $=0.91 \pm 0.045, \mathrm{p}=0.036$ ) and had blunted constrictor responses to LNMMA (Emax wt $=106.1 \pm 30.10$ vs hIGFREO $=47.7 \pm 9.87, \mathrm{p}=0.048$ ) indicating reduced basal $\mathrm{NO}$ bioavailability. In response to insulin EC from hIGFREO had: reduced NO release (wt $=4500 \pm 1000$ vs hIGFREO $=1500 \pm 700, p<0.05$ ); reduced eNOS activation (wt $=170 \% \pm 25$ vs hIGFREO $58 \% \pm 3, p<0.04$ ); and decreased phosphorylation of eNOS $(p=0.027)$. After endothelium denuding arterial injury hIGFREO mice demonstrated accelerated endothelium regeneration (recovered area: wt $=40.27 \% \pm 5.7$ vs hIGFREO $=57.25 \% \pm 2.3, p=0.003$ ) and enhanced endothelial cell migration under control conditions and in response to VEGF $(\mathrm{p}<0.001)$.

Conclusions Manipulation of IGF1-receptor numbers may represent a novel strategy for altering insulin sensitivity and vascular $\mathrm{NO}$ production. This data demonstrates uncoupling of endothelial $\mathrm{NO}$ bioavailability and vascular repair.

\section{LOCAL $\beta$-ADRENERGIC STIMULATION OVERCOMES SOURCE-SINK MISMATCH TO GENERATE FOCAL ARRHYTHMIA}

doi:10.1136/heartjnl-2012-301877b.110

${ }^{1} \mathrm{R} C$ Myles, ${ }^{* 2} \mathrm{~L}$ Wang, ${ }^{2} \mathrm{C}$ Kang, ${ }^{2} \mathrm{D} \mathrm{M}$ Bers, ${ }^{2} \mathrm{C}$ M Ripplinger. ${ }^{1}$ University of Glasgow, Glasgow, UK; ${ }^{2}$ University of California, Davis, California, USA

Background $\beta$-Adrenergic receptor ( $\beta$-AR) stimulation produces sarcoplasmic reticulum (SR) $\mathrm{Ca} 2+$ overload and delayed afterdepolarisations (DADs) in isolated ventricular myocytes. However, in the intact heart, strong electrotonic coupling means that depolarisation from many thousands of cells is required for an action potential to propagate. The mechanisms by which cellular DADs are synchronised to overcome the source-sink mismatch and produce focal arrhythmia remain unknown. We aimed to determine if localised $\beta$-AR stimulation can produce spatio-temporal synchronisation of $\mathrm{DADs}$ in the intact heart, and to examine the effects of tissue geometry and cell-cell coupling on the induction of focal arrhythmia.

Methods and Results Simultaneous optical mapping of transmembrane potential $(\mathrm{Vm})$ and $\mathrm{Ca} 2+$ transients was performed in normal rabbit hearts during subepicardial injections $(50 \mu \mathrm{l})$ of norepinephrine (NE, 30-250 $\mu \mathrm{M}$ ) or control (normal Tyrodes). The protocol was performed at baseline and during partial gap junction uncoupling with carbenoxolone (CBX). Local NE produced premature ventricular complexes (PVCs) arising from the application site in all 15 hearts, and a dose-response was evident (low-dose: $0.45 \pm 0.62$ vs high-dose: $1.33 \pm 1.46$ PVCs/application, $p<0.0001$ ) NE-induced PVCs demonstrated areas of abnormal Vm-Ca2+ delay at the initiation site, indicating a Ca2+-mediated mechanism. PVCs were more inducible with $\mathrm{NE}$ at RV vs LV injection sites $(1.48 \pm 1.50$ vs $0.55 \pm 0.89, \mathrm{p}<0.01)$ and following CBX $(2.18 \pm 1.43$ vs $1.33 \pm 1.46$, $\mathrm{p}<0.05)$. Analysis of $\mathrm{NE}$ tissue exposure and $\mathrm{Vm}-\mathrm{Ca} 2+$ dynamics revealed that differences in focal arrhythmia propensity between RV and LV, and following gap junction uncoupling were due to modulation of source-sink interactions.

Conclusions These data provide the first experimental demonstration that localised $\beta$-AR stimulation can produce spatiotemporal synchronisation of SR Ca2+ overload and release in the intact heart and highlight the critical nature of the source-sink balance in the initiation of focal arrhythmias.

\section{ARRHYTHMOGENIC RIGHT VENTRICULAR CARDIOMYOPATHY-LIKE PHENOTYPE REVEALED BY ENDURANCE TRAINING IN HETEROZYGEOUS DESMOGLEIN2 MUTANTS}

doi:10.1136/heartjnl-2012-301877b.111

${ }^{1} \mathrm{~L}$ Fabritz, ${ }^{2} \mathrm{~L}$ Fortmueller, ${ }^{2} \mathrm{D}$ Kucerova, ${ }^{2} \mathrm{~S}$ Sakhtivel, ${ }^{1} \mathrm{~F}$ Syeda, ${ }^{1} \mathrm{P}$ Kirchhof, ${ }^{3} \mathrm{R}$ E Leube, ${ }^{3} \mathrm{C}$ Krusche. ${ }^{1}$ Centre for Cardiovascular Sciences, University of Birmingham, Birmingham, UK; ${ }^{2}$ Department of Cardiology, University Hospital Muenster, Muenster, Germany; ${ }^{3}$ Institute of Molecular and Cellular Anatomy, RWTH Aachen University, Aachen, Germany

Background Arrhythmogenic right ventricular cardiomyopathy (ARVC) is a rare cardiomyopathy but significantly contributes to sudden cardiac death in young otherwise healthy patients, especially long durance athletes. $5 \%-10 \%$ of patients with ARVC harbour mutations in the extracellular domains of the desmoglein (DSG) 2 gene. To assess the role of DSG2 in the ARVC pathomechanism, mice lacking exons 4-6 of the endogenous DSG2 gene (DSG2mt) were generated. Homozygous DSG2 $\mathrm{mt} / \mathrm{mt}$ mice developed dilatation of ventricles and pronounced fibrosis. Heterozygous DSG2mt/ wt mutants, however, did not show such morphological alterations. Objective To study whether physical exercise provokes a cardiac phenotype in DSG2wt/mt mice they were subjected to endurance training together with wild-type (WT) littermates.

Methods/Results Group swimming training sessions were performed 6 times a week starting with 5 min and gradually incrementing to $90 \mathrm{~min} / \mathrm{d}$ for 7 weeks. Echocardiography was performed before and after training using a small animal ultrasound unit. Right ventricular (RV) diameters were increased in DSG2wt/mt both compared to pretraining and compared to WT after training. Right ventricular function was also decreased after training compared to pretraining and compared to WT littermates (see Abstract 111 table 1 for values, ${ }^{*} \mathrm{p}<0.05, \mathrm{~d}=$ diastolic, $\mathrm{s}=$ systolic, lav $=$ long, sav $=$ short axis view, $\mathrm{FAC}=$ fractional area shortening, $\mathrm{HR}=$ heart rate). Neither left ventricular diameters nor function differed between DSG2wt/ 